\title{
Pressure-induced high-spin to low-spin transition in FeS evidenced by x-ray emission spectroscopy
}

\author{
J.-P. Rueff, C.-C. Kao \\ National Synchrotron Light Source, Brookhaven National Laboratory, Upton, NY 11973, USA \\ V. V. Struzhkin, J. Badro, J. Shu, R. J. Hemley, and H. K. Mao \\ Geophysical Laboratory and Center for High Pressure Research, Carnegie Institution of Washington, \\ 5251 Broad Branch Road NW, Washington, DC 20015, USA
}

\begin{abstract}
We report the observation of the pressure-induced high-spin to low-spin transition in FeS using new high-pressure synchrotron x-ray emission spectroscopy techniques. The transition is evidenced by the disappearance of the low-energy satellite in the Fe $\mathrm{K} \beta$ emission spectrum of FeS. Moreover, the phase transition is reversible and closely related to the structural phase transition from a manganese phosphide-like phase to a monoclinic phase. The study opens new opportunities for investigating the electronic properties of materials under pressure.
\end{abstract}

The study of the electronic structure of highly correlated transition metal compounds has been an important subject in condensed-matter physics over the last several decades. The theoretical phase diagram proposed by Zaanen, Sawatzky, and Allen [1] is one of the key steps leading to a better understanding of the materials. In addition to the on-site $d$ - $d$ Coulomb interaction $(U)$ employed in the original Mott-Hubbard theory, the ligand-valence band width $(W)$, the ligand-tometal charge-transfer energy $(\Delta)$, and the ligand-metal hybridization interaction $(T)$ are explicitly included as parameters in the model Hamiltonian. This classification scheme has been very successful in describing the diverse properties and some seemingly contradicting behavior of a large number of these compounds. However, these highenergy-scale charge fluctuations are primarily characteristic of the elements involved, and thus cannot be freely adjusted for systematic study of their effects, although they can be varied somewhat by external temperature and magnetic field. On the other hand, pressure can introduce much larger perturbations of these parameters than can either temperature or magnetic field. Hence, it is of great interest to study the high-pressure behavior of these systems, and specifically, to correlate observed transformations with changes in electronic structure.

Unfortunately, high-pressure electronic structure studies have been severely handicapped because only a few standard spectroscopic techniques are compatible with high-pressure cells. Despite rapid developments in synchrotron-based x-ray spectroscopy, there have been limited investigations at high pressure beyond a few $\mathrm{x}$ ray absorption studies that have focused mainly on local atomic coordination and structure [2]. This limited study is due to the strong attenuation of x-ray photons below $10 \mathrm{keV}$ through the pressure cell (e.g., diamond anvils), the energy range that covers all the $3 d$ transition element $\mathrm{K}$ absorption edges, the $4 f$ rare earth $\mathrm{L}$ absorption edges, and the $5 f$ actinides $\mathrm{M}$ absorption edges, as well as their characteristic emission lines. This problem can be overcome by the use of beryllium as a gasket material in diamond anvil cells [3], and allowing both the incident and transmitted/scattered photons to pass through the low absorbing Be gasket instead of diamond. With this technique, the usable x-ray energy range can in principle be extended to include the important spectral region from 3 to $10 \mathrm{keV}$, making possible a wide variety of $\mathrm{x}$ ray spectroscopic studies of the electronic structure of materials under pressure.

Here we present a study of the high-spin to low-spin (HSLS) transition in FeS using new high-pressure, highresolution x-ray emission spectroscopy (XES) techniques. Under ambient pressure, $\mathrm{FeS}$ is an anti-ferromagnetic insulator $\left(\mathrm{T}_{N}=598 \mathrm{~K}\right)$, 4.57 and has a NiAs-related (troilite) structure. More importantly, FeS falls at the boundary between charge-transfer and Mott-Hubbard insulators in the ZSA phase diagram $(\Delta<U$ and $U$ relatively small [6]), so that its electronic structure is far more complicated, and has been very controversial. Pressure-induced structural phase transitions in FeS have been extensively studied, in part because the material is considered to be a major component of the cores of terrestrial planets [17 12]. $\mathrm{FeS}$ undergoes two structural phase transitions at ambient temperature, from the NiAs-related to a MnP-related structure at $3.5 \mathrm{GPa}$, and then to a monoclinic phase at 6.5 GPa. The latter transition is further accompanied by an abrupt shortening of the $c$ parameter from $5.70 \AA$ to $5.54 \AA$ [7]. Conventional Mössbauer spectroscopy experiments 13, 14 have shown that the structural transition at $6.5 \mathrm{GPa}$ is accompanied by the disappearance of the magnetic moment-induced hyperfine splitting. Resistivity measurements also hinted a possible insulator to semimetal transition at $3.5 \mathrm{GPa}$, and perhaps even a metallic phase above $7 \mathrm{GPa}$ 15. We report the observation of a HS-LS transition in FeS under pressure. The spin state 
of the Fe in FeS is monitored by high-resolution measurement of the $\mathrm{Fe} \mathrm{K} \beta$ emission line. The emission spectrum of HS Fe is characterized by a main peak with energy of $7058 \mathrm{eV}$, and a satellite peak located about $12 \mathrm{eV}$ lower in energy. When the pressure exceeds 6.9 GPa, the satellite vanishes, indicating the transition to the low spin-state with the collapse of the Fe $3 d$ magnetic moment.

The experiments were carried out at the X25 hybrid wiggler beamline at the National Synchrotron Light Source (NSLS), Brookhaven National Laboratory. The beamline consists of a Pt-coated double-focusing mirror located upstream of a two-crystal monochromator. Focused white beam was used to maximize the incident $\mathrm{x}$-ray flux for the excitation of the emission line. The typical beam size at the sample position was about 0.5 $\mathrm{mm}^{2}$. The spectrometer for the x-ray emission measurement is a 1-meter Rowland circle instrument laid out in the horizontal plane. A spherically-bent $\mathrm{Si}(333)$ singlecrystal analyzer crystal was used, with a Bragg angle corresponding to the $\mathrm{Fe} \mathrm{K} \beta$ emission line $(7058 \mathrm{eV})$ of $57.17^{\circ}$. Use of a $200 \mu \mathrm{m}$ slit in front of the detector produced an energy resolution of about $1.2 \mathrm{eV}$ at $7 \mathrm{keV}$. Two-dimensional scans were carried out to ensure accurately measured lineshapes [16]. The emission spectrum was then reconstructed by extracting the maximum of intensity in the detector scan at each Bragg angle. Typical collection times were about 90 minutes per spectrum.

High-purity FeS was loaded into a diamond cell together with 4:1 mixture of methanol-ethanol as pressure medium [17]. The diamond-cell gasket was initially a 5 $\mathrm{mm}$ diameter by $1 \mathrm{~mm}$ thick Be foil. The pressure was measured by the ruby fluorescence technique. In order to reduce background signal, the incident white beam was collimated by $150.50 \mu \mathrm{m}^{2}$ water-cooled $1 \mathrm{~mm}$ thick Ta slits. The slit assembly was in turn mounted on a watercooled copper block located in front of the cell, which was oriented vertically so that both the incident and collected $\mathrm{x}$-rays passed through the Be gasket. The emitted x-rays were detected at a $90^{\circ}$ scattering angle to further reduce background signal. To evaluate the signal from the Be gasket (known to contain small amount of Fe impurities), a spectrum measured $150 \mu \mathrm{m}$ from the sample was subtracted from that measured at the sample position.

Figure 1 shows the $\mathrm{Fe} \mathrm{K} \beta$ XES of FeS between ambient pressure and $11.5 \mathrm{GPa}$. All spectra show a main peak located at $7058 \mathrm{eV}$, usually referred to as the $\mathrm{K} \beta_{1,3}$ line. More importantly, a well-defined satellite located at $7045.5 \mathrm{eV}$, denoted $\mathrm{K} \beta^{\prime}$ in the following, appears only in spectra measured for pressures below 6.3 GPa. The satellite intensity disappears in spectra for pressures ranging between 6.3 and 11.5 GPa. The width of the main line also shows significant narrowing in this pressure range. The spectrum taken at $6.1 \mathrm{GPa}$ shows intermediate behavior between these two groups of spectra. It is probably due to the pressure gradient in the sample, which is estimated to be less than $0.5 \mathrm{GPa}$. Moreover, the ob- served changes are reversible as the pressure reduced back to ambient.

To illustrate the pressure-induced effects more clearly, the intensity of the satellite as a function of the applied pressure is shown in Fig. 22. The intensity of the 7045.5 $\mathrm{eV}$ satellite of each spectrum was determined by subtracting the spectrum at $11.5 \mathrm{GPa}$ (with no satellite) and fitting the resulting profile with two Voigt functions (at 7045.5 and $7056 \mathrm{eV}$ ). The difference spectra are shown in the inset of Fig. 2. In addition to the satellite peak in the difference spectra, a peak around $7056 \mathrm{eV}$ is observed, which is also a signature of the HS state (as discussed below). Although the position and intensity of this peak are subject to some uncertainty due to the subtraction procedure, the peak disappears around $7 \mathrm{GPa}$ (like the 7045.5 eV satellite). From Fig. 2, a reversible transition occurs between 6.0 GPa and 7.0 GPa. Since the uncertainty in pressure due to the relaxation processes and the pressure gradient in the cell is about $0.5 \mathrm{GPa}$, the observed change thus coincides with the pressure of $6.5 \mathrm{GPa}$ at which the magnetic splitting disappeared in the Mössbauer measurement. Notably, there is no appreciable change in the satellite intensity at $3.5 \mathrm{GPa}$, the pressure corresponding to the NiAs- to MnP-related structural phase transition.

To interpret the observed spectral changes, a brief review of the theory of $\mathrm{K} \beta$ x-ray emission spectra is given. The $\mathrm{K} \beta$ emission lines are characteristic $\mathrm{x}$-ray lines originating from the $3 p \rightarrow 1 s$ decay. For a large number of transition metal compounds, the $\mathrm{K} \beta$ spectra has been interpreted using atomic multiplet calculations and configuration interaction. It is now widely accepted that the spectral shape of $\mathrm{K} \beta$ emission line for these compounds is dominated by final state interaction between the $3 p$ core hole and the electrons of the partially filled $3 d$ shell. Qualitatively, the main effect is due to the exchange interaction between the core hole and the local moment, which results in splitting of the $\mathrm{K} \beta$ spectrum into HS and LS final states. This simple picture also predicts that the energy separation between the two peaks is given by the product of the exchange integral $J$ and $(2 S+1)$, where $S$ is the total spin of the $3 \mathrm{~d}$ shell; and that the intensity ratio between the two is given by $S /(S+1)$ [18]. Both the energy splitting and intensity ratio are modified when configuration interaction is taken into account [19]. These calculations show that the $3 p^{\uparrow} 3 d^{\uparrow}$ final state is characterized by a single peak that constitutes most of the intensity of the main emission line. On the other hand, the $3 p^{\downarrow} 3 d^{\uparrow}$ final state is further split into two components, one at significantly lower energy than predicted by simple theory and one at slightly lower energy than the main emission line $\left(3 p^{\uparrow} 3 d^{\uparrow}\right)$ which appears as a shoulder. However, the simplified picture does point out the qualitative changes expected for the $3 d$ electrons going from HS to LS states, namely smaller energy splitting between the main peak and the satellite as well as a reduction in the satellite to main peak intensity ratio. 
The above theoretical approach has been successfully applied to the interpretation of the $\mathrm{K} \beta$ emission spectra of a number of Mn 20 22, and Fe 23, 24 compounds recently. The sensitivity of the $\mathrm{K} \beta_{1,3}$ emission lineshape to the spin state of the system is demonstrated Fig. 3, where the $\mathrm{Fe} \mathrm{K} \beta_{1,3}$ line from $\mathrm{FeO}$ and $\mathrm{FeS}_{2}$ are shown. These spectra were recorded at ambient pressure, and the nominal oxidation state is +2 for Fe in all three compounds; however, $\mathrm{Fe}$ is in the HS state in $\mathrm{FeO}$ and in the LS state in $\mathrm{FeS}_{2}$. Note the pronounced satellite structure in the $\mathrm{K} \beta$ spectrum of $\mathrm{FeO}$, and the lack of a satellite feature for $\mathrm{FeS}_{2}$. Detailed inspection shows that there is a small shoulder on the low energy side of the main peak for $\mathrm{FeO}$, indicating the presence of the small HS component discussed above. On the other hand, the main peak for $\mathrm{FeS}_{2}$ is narrower and more symmetric, suggesting that in addition to the disappearance of the low energy satellite, the higher energy HS component is absent as well. These two examples clearly show that the spectral shape of the $\mathrm{K} \beta$ emission line can be used to characterize the spin state of the transition-metal ions in these systems.

We can now describe more precisely the pressure induced electronic transition in FeS. Under ambient condition, $\mathrm{Fe}$ in $\mathrm{FeS}$ is considered to be divalent with a HS electron configuration $\left(t_{2 g \uparrow}^{3} e_{g \uparrow}^{2} t_{2 g \downarrow}^{1}\right)$. This result is now well established through both Mössbauer spectroscopy and magnetic susceptibility measurements 14. The Mössbauer spectra clearly indicate divalent Fe. The isomer shift of $+0.76 \mathrm{~mm} / \mathrm{s}$ found in $\mathrm{FeS}$ is consistent with $\mathrm{Fe}^{2+}$ in the HS configuration, which is further confirmed by the large effective moment (estimated to be $5.5 \mu_{B}$ by magnetic susceptibility measurements [ 1 ]). In the LS state $\left(t_{2 g \uparrow}^{3} t_{2 g \downarrow}^{3}\right)$, the two final states of the $\mathrm{K} \beta$ emission line, $3 p^{\uparrow \downarrow} 3 d$, are degenerate because of the absence of a $3 d$ magnetic moment, thus the XES spectrum reduces to a single peak as observed.

The result presented here is also consistent with the disappearance of the magnetic splitting of the hyperfine field above $6.5 \mathrm{GPa}$ ( $c f$. Ref. [14]). It should be noted that Mössbauer spectroscopy cannot unambiguously discriminate between a local diamagnetic or paramagnetic state. The HS-LS transition may also account for the shrinkage of the lattice cell along the $c$ axis associated with the structural transition observed around $7 \mathrm{GPa}$. Indeed, it has been proposed that the spin-pairing on the Fe $3 d$ shell might cause a diminution of the Fe radius [11 and drives the observed transition.

Metallization is a competing process that could also lead to the pressure-induced reduction of the satellite amplitude. However, metallization alone cannot explain the disappearance of the satellite above the transition pressure since a well defined satellite also appears in the $\mathrm{K} \beta$ emission line of pure Fe-metal 25] whose position and intensity is comparable to the one found in FeS at low pressure. Also, if metallization only were to occur, one would expect the satellite intensity of metallized highspin $\mathrm{FeS}$ to be much stronger than that of metallic iron (the magnetic moments of high-spin FeS and metallic Fe are 5.5 and $2.2 \mu_{B}$ respectively), which is not consistent with our measurements.

Whether or not the observed transformation is coupled to a metal-insulator transition [26] is still an open question for both experiment and theory. Notably, the sequence of transitions (e.g., HS-LS, metallization, Mott transitions) in such materials generally is the subject of much current theoretical study [27].

High-pressure measurements of the valence band emission and resonant inelastic $\mathrm{x}$-ray scattering are in progress to clarify these issues. X-ray emission spectroscopy can also be extended to measure symmetryprojected local density of states of the valence band, which provides a direct measurement of the anion $p$-band bandwidth in transition-metal compounds. Pressure dependent measurements of the anion bandwidth would be important in determining the origin of metal-insulator transitions in these systems 28].

We thank N. Boctor for sample synthesis and characterization, and L. Berman, Z. Yin, and J. Hu for help with the experiments. This work was supported by the NSF and DOE (Contract No. DE-AC02-76CH00016 to NSLS).

[1] J. Zaanen, G.A. Sawatzky, and J.W. Allen, Phys. Rev. Lett. 55, 418 (1985).

[2] J.-P. Itié, A. Polian, D. Martinez, V. Briois, A. DiCicco, A. Philipponi, and A. San Miguel, J. de Physique IV. 7:C2, 31 (1997)

[3] R.J. Hemley, H.K. Mao, G. Shen, J. Badro, P. Gillet, M. Hanfland, and D. Haüsermann, Science 276, 1242 (1997).

[4] J.L. Horwood, M.G. Townsend, and H. Webster, J. Solid State Chem. 17, 35 (1976).

[5] K. Shimada et al., J. Electron Spectrosc. Relat. Phenom. 78, 317 (1996).

[6] A.E. Bocquet, T. Mizokawa, T. Saitoh, H. Namatame, and A. Fujimori, Phys. Rev. B 46, 3771 (1992).

[7] Y. Fei, T. Prewitt, H.K. Mao, and C.M. Bertka, Science 268, 1892 (1995).

[8] D. Sherman, Earth Planet. Sci. Lett. 132, 87 (1995).

[9] T. Kamimura, M. Sato, H. Takahashi, N. Mori, H. Yoshida, and T. Kaneko, J. Magn. Magn. Mater. 104107, 255 (1992).

[10] H.E. King and C.T. Prewitt, Acta Crystallogr. B 38, 1877 (1982).

[11] K. Kusaba, Y. Syono, T. Kikegawa, and O. Shimomura, J. Phys. Chem. Solids 58, 241 (1997).

[12] L.A. Taylor and H.K. Mao, Science 170, 850 (1970).

[13] H.E. King, D. Virgo, and H.K. Mao, Carnegi Inst. Washington Year Book 77, 830 (1978). 
[14] H. Kobayashi et al., J. Phys. Condens. Matter 9, 515 (1997).

[15] S. Minomura and H.G. Drickamer, J. Appl. Phys. 37, 3043 (1963).

[16] For the relatively low Bragg angles used in these measurements, the accurate tracking of analyzer and detector was crucial because misalignment between the two can cause an apparent reduction in intensity, thereby modifying the measured emission lineshape. For each Bragg angle, the detector was scanned over a range of $600 \mu \mathrm{m}$.

[17] Pure polycrystalline FeS was synthesized from highpurity Fe metal and sulfur (both $99.999^{+} \%$ ) in evacuated silica tubes. It was annealed at $675 \mathrm{~K}$ for several days until all of the sulfur reacted with the iron. The tubes were then transferred to $1275 \mathrm{~K}$ and annealed for one week before quenching rapidly in an ice-water bath. The homogeneity and purity of the sample was confirmed by electron microprobe analysis. A slight excess of iron was included to remain in the troilite side of the phase diagram and to insure that the material is stoichiometric.

[18] K. Tsutsumi, H. Nakamori, and K. Ichikawa, Phys. Rev. B 13, 929 (1976).

[19] B. Hermsmeier, C.S. Fadley, M.O. Krause, J. JimenezMier, P. Gerard, and S.T. Manson, Phys. Rev. Lett. 61, 2592 (1988).

[20] F.M.F de Groot, S. Pizzini, A. Fontaine, K. Hämäläinen, C.C. Kao, and J.B. Hastings, Phys. Rev. B 51, 1045 (1995).

[21] G. Peng et al., J. Am. Chem. Soc. 116, 2914 (1994).

[22] M. Taguchi, T. Uozumi, and A. Kotani, J. Phys. Soc. Jpn. 66, 247 (1997).

[23] G. Peng, X. Wang, C.R. Randall, J.A. Moore, and S.P. Cramer, Appl. Phys. Lett. 65, 2527 (1994).

[24] X. Wang, F.M.F. de Groot, and S.P. Cramer, Phys. Rev. B 56, 4553 (1997).

[25] G. Hölzer, M. Fritsch, M. Deutsch, J. Härtwig, and E. Förster, Phys. Rev. A 56, 4554 (1997).

[26] Although possible metallization was suggested in Ref. 14, only a slight increase in resistivity with increasing temperature was measured.

[27] R.E. Cohen, Y. Fei, R.T. Downs, I.I. Mazin, and D.G. Isaak, in High-Pressure Material Research, Materials Research Society Symposium Proceedings 499, 27 (1997).

[28] A.L. Chen, P.Y. Yu, and R.D. Taylor, Phys. Rev. Lett. 71, 4011 (1993).

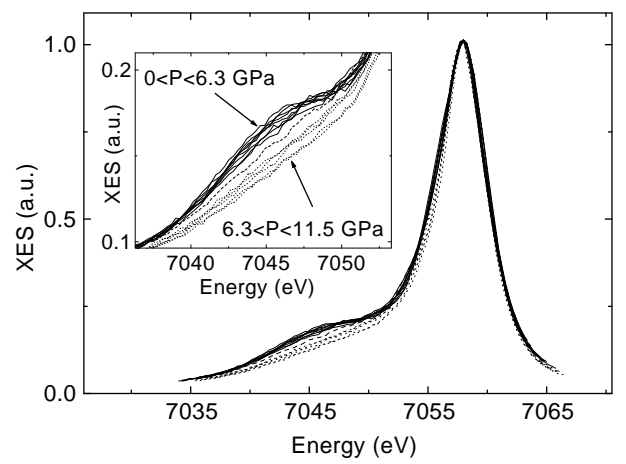

FIG. 1. Fe XES in FeS as function of pressure. The spectra were normalized with the intensity of the main peak set to unity, and aligned so that the (pressure independent) energy of the main peak is set to $7058 \mathrm{eV}$. At low pressure, the satellite at $7045.5 \mathrm{eV}$ is characteristic of the high-spin state (solid line) whereas the absence of satellite at high pressure denotes the transition to the low-spin state (dotted lines). The dashed line shows an intermediate spectrum obtained on decompression at $6.1 \mathrm{GPa}$. The sequence of pressures was 1.25, 2.0, 2.7, $3.5,6.3,7.5,10.0,11.5,8.2,6.9,6.1,5.2,1.4$, and $0.2 \mathrm{GPa}$.

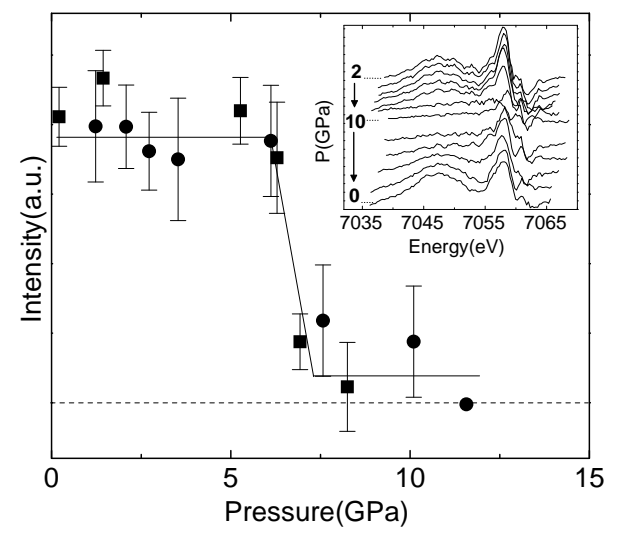

FIG. 2. Integrated intensity of the structure in the satellite region of the spectra shown in Fig. 1 obtained from a Voigt profile fitted to the difference spectrum upon compression (circles) and decompression (squares). The intensity was calculated from the difference spectra obtained by subtracting the $11.5 \mathrm{GPa}$ spectrum from each scan (inset). The pressure in the inset follows from top to bottom the loop as indicated in the caption to Fig. 1 and given on the left-hand side of the inset. The second peak in $7056 \mathrm{eV}$ region is distorted by the subtraction procedure; consequently its intensity is not shown. Spurious peaks in the $\mathrm{K} \beta_{1,3}$ shoulder at 7058 to 7062 $\mathrm{eV}$ arise from uncertainties associated with the subtraction procedure. The solid line is a guide to the eye; the dashed line shows zero intensity level.

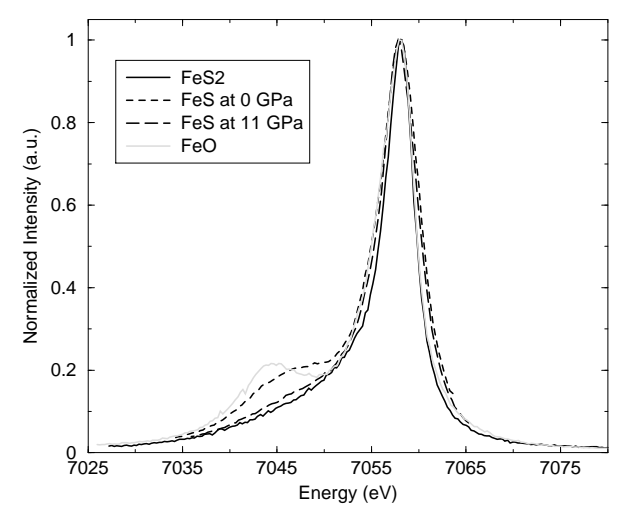

FIG. 3. XES spectra of FeS in the high-spin (room pressure) and low-spin (11.5 GPa) states, along with room pressure reference spectra of two iron compounds with +2 oxidation states for iron, namely $\mathrm{FeO}$ (HS) and $\mathrm{FeS}_{2}$ (LS). The difference between the low-spin spectra of $\mathrm{FeS}$ and $\mathrm{FeS}_{2}$ is within the measured error. 